\title{
FRACTAL PROPERTIES OF EXTRAGALACTIC JETS: EVIDENCE OF TURBULENCE?
}

\author{
G. BODO, S. MASSAGLIA \\ Osservatorio Astronomico di Torino \\ Strada dell'Osservatorio 20, 10025 Pino Torinese, Italy
}

L. FERETTI

Istituto di Radioastronomia del CNR
Via Gobetti 101, I-40129 Bologna, Italy

AND

A. FERRARI, D. DALL'ANESE

Dipartimento di Fisica Generale, Università di Torino Via P. Giuria 1, I-10125 Torino, Italy

Several different properties of extragalactic radio sources have been attributed to the effects of turbulence. The morphological appearance of FRI sources has been often interpreted as the result of turbulent entrainment in subsonic or transonic flows (Bicknell 1984, 1986). Moreover, particle acceleration by MHD turbulence via a second order Fermi process is one of the possible ways for accelerating the synchrotron emitting relativistic particles (see Ferrari, Trussoni \& Zaninetti 1979). Turbulence appears therefore as an important ingredient in the theoretical modelling of extragalactic radio sources; however, we do not have, unfortunately, any direct evidence of it.

Many recent works on turbulence have analysed the geometrical properties of different kinds of surfaces in turbulent flows. For example, introducing a tracer (a passive scalar) in such a flow, the surface, known as "scalar interface" separating the "coloured" fluid from the "non-coloured" one, acquires fractal properties when advected by turbulent motions. Measures of its fractal dimension $D$ give a value of $D=2.35 \pm 0.05$ ( $D-1$ for sections), irrespective of the type of flow or of the flow conditions. Similar values have been also obtained for different kinds of surfaces and in different contexts. We recall that the fractal dimension $D$ is defined as $N=r^{-D}$, with $N$ the number of objects with characteristic linear dimension larger than $r$. 

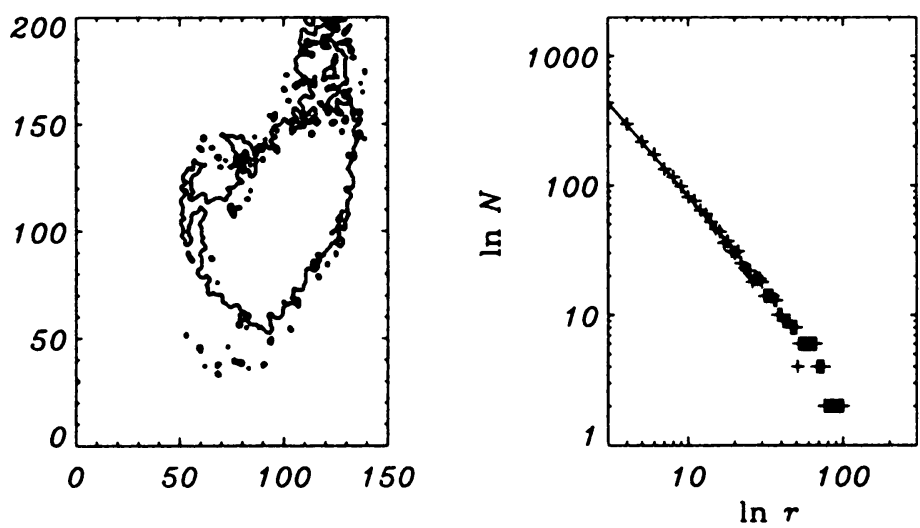

Figure 1. Brightness contour at $3 \sigma$ level $\left(1.1 \times 10^{-4} \mathrm{Jy} /\right.$ beam $)$ of the southern lobe of 3C449 (left panel); $\ln N$ vs $\ln r$ curve yielding $D=1.39$ (right panel)

We have analyzed the fractal properties of the boundaries of the brightness distribution (using thresholds corresponding both to $3 \sigma$ and $5 \sigma$ levels) in the VLA radio maps of 3 FRI sources: $3 \mathrm{C} 449$, 3C465, $4 \mathrm{C} 29.30$. We report in Fig. 1 and Table 1 the results obtained.

TABLE 1. Fractal Dimensions

\begin{tabular}{lll}
\hline Source & $3 \sigma$ level & $5 \sigma$ level \\
\hline $3 \mathrm{C} 449$ & 1.36 & 1.21 \\
$3 \mathrm{C} 465$ & 1.19 & 1.28 \\
$4 \mathrm{C} 29.30$ & 1.17 & 1.13 \\
\hline
\end{tabular}

We have also measured the fractal dimension of the scalar interface in the results of numerical simulations of supersonic fluid jets in 2-D (slabs), undergoing Kelvin-Helmholtz instabilities. We can follow there the evolution towards a turbulent state, with $D$ increasing from unity, in the initial state, to $D \simeq 1.35$, in the turbulent state.

\section{References}

Bicknell G.V. (1984) ApJ, 286, 68

Bicknell G.V. (1986) ApJ, 300, 591

Ferrari A., Trussoni E., Zaninetti L. (1979) $A \& A, 79,190$ 